\title{
Generating Better Medicines for Cancer
}

\author{
Stuart S. Dunn ${ }^{\dagger, \perp}$, James D. Byrne ${ }^{\ddagger, \perp}$, Jillian L. Perry ${ }^{\S}$ Kai Chen ${ }^{\circ}$, and Joseph M. \\ DeSimone ${ }^{\ddagger, \S,}{ }^{\circ}, \square, \Delta,{ }^{\wedge}, \infty, \cap, \Delta, \#,{ }^{*}$ \\ tWyss Institute for Biologically Inspired Engineering, School of Engineering and Applied \\ Sciences, Harvard University, Cambridge, Massachusetts 02138, USA
}

‡Eshelman School of Pharmacy, University of North Carolina, Chapel Hill, North Carolina 27599, USA

$\S$ Lineberger Comprehensive Cancer Center, University of North Carolina, Chapel Hill, North Carolina 27599, USA

${ }^{\circ}$ Department of Chemistry, University of North Carolina, Chapel Hill, North Carolina 27599, USA

Department of Pharmacology, University of North Carolina, Chapel Hill, North Carolina 27599, USA

$\triangle$ Department of Biomedical Engineering, University of North Carolina, Chapel Hill, North Carolina 27599, USA

`Carolina Center of Cancer Nanotechnology Excellence, University of North Carolina, Chapel Hill, North Carolina 27599, USA

${ }^{\infty}$ Institute for Advanced Materials, University of North Carolina, Chapel Hill, North Carolina 27599, USA

Institute for Nanomedicine, University of North Carolina, Chapel Hill, North Carolina 27599, USA

$\triangle$ Department of Chemical and Biomolecular Engineering, North Carolina State University, Raleigh, North Carolina 27695, USA

\#Sloan-Kettering Institute for Cancer Research, Memorial Sloan-Kettering Cancer Center, 1275 York Avenue, New York, New York 10065, USA

\section{Abstract}

The complexity of tumor biology warrants tailored drug delivery for overcoming the major challenges faced by cancer therapies. The versatility of the PRINT ${ }^{\circledR}$ (Particle Replication In Nonwetting Templates) process has enabled the preparation of shape- and size-specific particles with a wide range of chemical compositions and therapeutic cargos. Different particle matrices and drugs may be combined in a plug-and-play approach, such that physico-chemical characteristics of delivery vectors may be optimized for biocompatibility, cargo stability and release, circulation half-life, and efficacy. Thus, the engineering of particles for cancer therapy with specific biophysical behaviors and cellular responses has been demonstrated via the PRINT process.

*Corresponding author: desimone@unc.edu.

$\perp_{\text {Author Contributions }}$

These authors contributed equally to this work.

The authors declare competing financial interest. Joseph DeSimone is a founder, member of the board of directors, and maintains a financial interest in Liquidia Technologies. Liquidia was founded in 2004 to commercialize PRINT technology and other discoveries of Professor Joseph DeSimone and colleagues at the University of North Carolina, Chapel Hill. 


\section{Keywords}

PRINT; soft lithography; nanoparticle; cancer; medicine; drug delivery

\section{Increasing the Efficacy of Particle-Based Therapeutics by Addressing Tumor Biology}

The biggest challenge of nanoparticles (NPs) for the treatment of cancer is penetrating a tumor microenvironment that is spatially and temporally heterogeneous. Abnormal tumor vasculature, lack of functional lymphatics, and elevated interstitial fluid pressure (IFP) reduce the delivery of particles into tumors. ${ }^{1}$ Blood flow in tumors is highly irregular based upon the spatial distribution of the vasculature with wide interendothelial junctions, thin and thick basement membrane, large numbers of fenestrae, and transendothelial channels. Unperfused tumoral regions create a hostile tumor microenvironment (low partial pressure of oxygen, low $\mathrm{pH}$, and necrotic tissue) that can lead to drug resistance and tumor progression (Figure 1). ${ }^{1}$ There are some primary tumors that are very poorly vascularized, such as pancreatic cancer, and the hypovascular nature of these tumors limits the ability to deliver therapeutics in a highly efficient manner. ${ }^{2}$ Furthermore, the interstitial fluid pressure (IFP) is uniformly elevated in tumors due to the combination of highly permeable tumor vasculature and the lack of functional lymphatic tissue in the tumor interstitial space, which limits drug transport into the center of the tumor. ${ }^{3}$

The tumor characteristics that create a barrier for particle delivery into tumors are the same ones that allow for effective particle transport into the tumor. Nanoparticles can extravasate into tumor tissue from large pores that are present in the tumor vasculature and be retained within the tumor due to the high IFP; this is known as the enhanced permeation and retention (EPR) effect. Thus, the EPR effect is utilized as a passive targeting method for delivery of NPs into tumors. Tumor penetration also requires a long circulation half-life to allow for extravasation of the particle across the hyper-permeable tumor vessels and effective diffusion through the tumor interstitial space. ${ }^{4}$

The PRINT process ${ }^{5}$ is capable of controlling particle size, shape, surface chemistry, modulus, matrix, and drug loading. The major barriers for nanoparticle intratumoral transport may therefore be overcome by tuning the particle physico-chemical characteristics. For example, a pH-sensitive matrix can be incorporated into the PRINT process for fabricating particles capable of selective drug release within the acidic tumor microenvironment and intracellular endosome. ${ }^{6}$ Additionally, highly flexible particles with particular dimensions can be fabricated using the PRINT process to improve transport through the tumor extracellular matrix. ${ }^{7}$ The dimensions of PRINT particles range from sub-100 $\mathrm{nm}$ to micron-scale sizes with unique shapes such as pollen, toroids, trapezoids, helicopters, lollipops, cylinders, filamentous particles, and red blood cell mimics. Molecular conjugates and NPs prepared through other fabrication techniques, e.g. metal NPs, may adopt smaller sizes than conventional PRINT particles; however, sub-20 nm features have been replicated. ${ }^{8}$

\section{Extending Circulation Times and Enabling Active Targeting by Modifying the Particle Surface}

Upon contact with the blood stream, the surface of the nanoparticles can become coated with blood proteins that essentially mark the NPs for clearance from the body. This process is known as opsonization. In an effort to improve circulation half-life by reducing NP interactions with blood components and MPS (mononuclear phagocyte system) cells, 
poly(ethylene glycol) (PEG) has been extensively used on a variety of NP systems. ${ }^{9}$ There is a general consensus that stealth properties can be achieved by coating NPs with a high density of PEG of molecular weights ranging from $2 \mathrm{kDa}$ to $10 \mathrm{kDa} .{ }^{9}, 10$ Studies have shown that these stealth properties not only decrease NP elimination by the MPS system, they increase passive tumor targeting through the EPR effect. ${ }^{11}$ There are a number of passively targeted PEGylated polymeric NPs in early phase clinical trials for treating a variety of cancers. ${ }^{12}$ These NPs have clearly demonstrated wider therapeutic windows and lower systemic toxicities. It is important to note that these passive targeting strategies only increase accumulation of the drug at the tumor site; however, this extracellular delivery strategy is less effective for NPs or drugs that are not readily taken up by cancer cells. Therefore, much effort is now focused on investigating actively targeted NPs to increase specific cell uptake through receptor-mediated endocytosis. ${ }^{12,13}$

Active targeting uses affinity ligands to direct the binding of NPs to receptors overexpressed on diseased tissues and/or cells. These ligands can be antibodies, aptamers, proteins, peptides, or small molecules. ${ }^{12,13}$ Methods for preparing these actively targeted NPs involve either conjugation of targeting ligands to the surface of the NP or through self-assembly using prefunctionalized copolymers. ${ }^{12,13}$ Though the platform for an actively targeted NP was described more than 30 years ago, only a few targeted NPs have entered clinical trials. ${ }^{12}$ The key challenge for this platform is the ability to balance physico-chemical parameters that simultaneously confer targeting, stealth properties, and controlled drug release. It has been demonstrated that as the surface density of ligands is increased, NP surfaces become less stealth-like, which results in rapid clearance from the blood stream and poor EPR. ${ }^{13}$ Therefore, having the ability to narrowly optimize surface densities of stealth PEGs and targeting ligands on a NP is essential for in vivo success and clinical translation.

We demonstrated the ability to precisely control PEG density on the surface of hydrogel PRINT NPs, ranging from non-PEGylated to PEG mushroom and PEG brush decorated NPs. Increasing the PEG density on NPs significantly reduced protein adsorption and macrophage association, and increased circulation half-life. ${ }^{15}$

We have also shown that PRINT NPs conjugated with human transferrin or transferrin receptor monoclonal antibody can specifically target a broad spectrum of cancers, making it a useful platform for targeted drug delivery. ${ }^{16}$ Moreover, an essentially nontoxic transferrin and transferrin receptor antibody OKT9 was converted into a potential cancer therapeutic by harnessing the multivalent effect through conjugation to PRINT NPs. ${ }^{16}$ As stated above, having the ability to optimize NP physico-chemical properties is essential for in vivo success. The PRINT process allows for precise control over surface densities of both stealth PEG and targeting ligands on NPs, which will enable the optimization of passive and active targeting for enhanced drug delivery. The density of ligands conjugated to cylindrical (diameter $[d]=200 \mathrm{~nm}$; height $[h]=200 \mathrm{~nm})$ or rice-shaped $(d=80 \mathrm{~nm} ; h=320 \mathrm{~nm}) \mathrm{NPs}$ was quantified through spectrophotometric measurements for PEGs (using fluorescent PEGs) and BCA assays for proteins. By controlling the stoichiometric ratio of reactive PEGs to nanoparticles, the density of ligands was tuned accordingly. ${ }^{15}$

\section{Encapsulating a Wide Variety of Cargos using Different Particle Matrices}

The top down fabrication of particles via the PRINT process utilizes chemically resistant perfluoropolyether-based molds, which allow for the composition to be dictated in a plugand-play manner. Inherent solubility issues, e.g. those between hydrophobic and hydrophilic molecules, may minimize the accessibility of neat particle formulations across all compositions; however, additives may be implemented to aid in homogenization of certain formulations. The composition of PRINT particles may be selected for compatibility, 
stability, and efficacy in a given application for the therapeutic cargo encapsulated. Particle matrices composed of natural and synthetic polymers, pure drugs, and crosslinked networks have been explored and designed with features for effective delivery (Figure 2). Molds can be filled with pre-particle components through lamination of a solution or film followed by solidification (e.g. photochemical curing).

Through the plug-and-play approach enabled by the PRINT process, chemotherapeutics have been loaded into reductively-labile hydrogel particles, biodegradable polyester particles, and hydrogel NPs. Doxorubicin, a potent DNA intercalating chemotherapy, was physically encapsulated in PEG hydrogel particles, prepared by UV-curing molds filled with a pre-particle solution containing a degradable crosslinker, photoinitiator, and functional acrylics. ${ }^{17}$ Amorphous drug-polymer PRINT particles consisting of docetaxel, an antimitotic chemotherapy, and poly(lactide-co-glycolide) (PLGA) achieved encapsulation efficiencies greater than $90 \%$ and drug loadings up to 40 weight percentage (wt $\%$ ). ${ }^{18}$ Three chemotherapeutics bearing pendant alcohols were derivatized as prodrugs with acrylates containing acid-labile silyl ether linkages for incorporation into cationic PEG-based hydrogel NPs. ${ }^{19}$

Small interfering RNA (siRNA) oligonucleotides have been loaded into PLGA and hydrogel NPs through physical entrapment and reversible covalent incorporation. For PLGA, the molecular weight and lactide:glycolide ratio influenced the encapsulation efficiency of siRNA, reaching $46 \%$ in $80 \times 320 \mathrm{~nm}$ rod-shaped NPs. ${ }^{20}$ For hydrogel NPs, a delivery sheet of siRNA (in native or macromer form) and hydrophilic acrylate precursors (cationic, fluorescent, and PEGs) were married to PRINT molds through lamination followed by photochemical curing to provide electrostatic or covalent association of siRNA with NPs. ${ }^{21}$ In addition, RNA Replicon, an autonomously replicating RNA, has been loaded into bovine serum albumin (BSA) protein-based particles for the development of vaccines. ${ }^{22}$ BSA-based particles were loaded at $1 \mathrm{wt} \%$ with RNA Replicon, encoding a protein detectable through ELISA. Proteins have also been loaded into extremely deformable hydrogel microparticles for the purpose of artificial blood. Hemoglobin was stably and uniformly loaded into red blood cell mimicking particles by covalent conjugation. ${ }^{23}$ High loadings of hemoglobin were achieved in microgels with preservation of protein activity and particle deformability. Enhanced efficacy of therapies could be realized by simultaneously loading multiple cargos into PRINT particles. For example, a chemotherapeutic and siRNAs targeting anti-apoptotic and angiogenic genes could be co-delivered to treat cancers.

\section{Controlling Release Profiles of Therapeutic Cargos for Particle-based Drug Delivery}

Controlled release of encapsulated therapeutics is crucial for improved efficacy and reduced toxicity. The basic principle for controlled release is to ensure minimal pre-mature release before reaching the site of activity but sufficient release after reaching the target, which can be specific tissues, cells or cellular compartments. While external stimuli including light, ultrasound, and magnetic fields have been applied to control release in other systems, PRINT particle delivery systems mainly utilize molecular design of the drug and particle matrix to synchronize the release profile with physiological conditions. Physiological changes, including $\mathrm{pH}$ gradient and redox states, have been utilized to trigger release of therapeutic cargos from PRINT particles at a desired site. ${ }^{19}$

The well-studied silyl ether protecting groups for hydroxyls have been incorporated into the particle matrix for creating acid-labile crosslinkers, ${ }^{19}$ for which the degradation profile can be easily tuned by varying bulkiness of alkyl substituent on the silicon atom. The amount and type of the silyl ether crosslinker incorporated in the particle composition determined 
the degradation rate of the particle matrices, allowing for control over the release rate of encapsulated therapeutics from hours to months. For drugs that are difficult to retain in intrinsically porous hydrogel particles, a silyl ether based pro-drug approach has been developed. The pro-drug can be polymerized into PRINT particles, releasing drugs at different rates depending on the silicon substituent and $\mathrm{pH}$. In vitro experiments demonstrated that particles could be fabricated to release drugs, like gemcitabine and camptothecin, rapidly and with comparable toxicities to the free drug. ${ }^{19}$

The reducing environment of the cytoplasm of cells can also be taken advantage of for controlled release of therapeutics. The high concentration of reduced glutathione in the cytoplasm, compared to the extracellular environment, can degrade the disulfide bond, which is widely used to fabricate degradable particles. ${ }^{22}$ The disulfide strategy has been applied to both hydrogel and protein-based PRINT particles. We demonstrated the reductive release of doxorubicin and resulting in vitro cell death in a cancer cell line from hydrogel PRINT particles crosslinked by a commercially available disulfide-containing crosslinker, while control particles crosslinked by a non-degradable crosslinker elicited no cytotoxicity. ${ }^{17}$ Similar to a pro-drug approach, siRNA was linked to an acrylate group through disulfide linkage and polymerized into hydrogel NPs. ${ }^{21}$ The siRNA cargo was found to be adequately protected by the particle matrix and only be released upon exposure to a reducing environment for effective gene knockdown in vitro compared to a nondegradable control siRNA macromer. In BSA-based PRINT particles loaded with RNA replicon, premature dissolution of protein was eliminated by incorporating a newly synthesized disulfide containing crosslinker. Bis(imidazole carboxylate) groups in the crosslinker reacted with amines in the protein to crosslink the particles, rendering them transiently insoluble. ${ }^{22}$ The crosslinked BSA particles preferentially dissolved under reducing conditions, with the dissolution rate controlled by adjusting the crosslinker concentration, and induced in vitro expression of protein.

Considering the abnormal vasculature and the prevalence of the EPR effect in tumors, delivery vectors with physico-chemical and geometric properties designed to reach diseased tissues should have high therapeutic indices and limited side effects. Bottom-up particle fabrication approaches can exhibit interdependent properties on particle size, shape, and surface chemistry; conversely, as a top-down fabrication technology, the PRINT platform offers the opportunity to independently control particle properties, which can be designed for delivery vectors to alleviate tumor burden and improve the quality of life for patients. Given the range of cargos, dimensions, compositions, and surface properties attained with PRINT particles, future biomedical endeavors may involve (1) exploring additional types of therapeutic cargos that face difficulties being incorporated into conventional delivery systems, (2) tuning vector dimensions, deformability, and composition to reach particular tissues, allow for specific transport behavior, and deliver therapeutics to cells effectively, (3) exploiting features of tissue environments, such as the presence of matrix metalloproteinases and cathepsins in cancers for degradation of particles or release of cargo, and (4) functionalizing the surface of particles with ligands to target endothelium or receptors on target cells.

\section{Acknowledgments}

This work was supported by Liquidia Technologies, the Carolina Center for Cancer Nanotechnology Excellence (U54CA151652), University Cancer Research Fund, NIH's Pioneer Award (IDP10D006432), R01 (R01EB009565), and National Science Foundation (DMR-0923604 and DMR-0923604).

\section{REFERENCES}

1. Jain RK, Stylianopoulos T. Nat. Rev. Clin. Oncol. 2010; 7:653-664. [PubMed: 20838415] 
2. Hidalgo M, Maitra A. New Engl. J. Med. 2009; 361:2094-2096. [PubMed: 19923581]

3. Jain RK, Tong RT, Munn LL. Cancer Res. 2007; 67:2720-2735. [PubMed: 17363593]

4. Pirollo KF, Chang EH. Trends Biotechnol. 2008; 26:552-558. [PubMed: 18722682]

5. Rolland JP, Maynor BW, Euliss LE, Exner AE, Denison GM, DeSimone JM. J. Am. Chem. Soc. 2005; 127:10096-10100. [PubMed: 16011375]

6. Parrott MC, Luft JC, Byrne JD, Fain JH, Napier ME, DeSimone JM. J. Am. Chem. Soc. 2010; 132:17928-17932. [PubMed: 21105720]

7. Merkel TJ, Jones SW, Herlihy KP, Kersey FR, Shields AR, Napier ME, Luft JC, Wug H, Zamboni WC, Wang AZ, Bear JE, DeSimone JM. Proc. Natl. Acad. Sci. U.S.A. 2011; 108:586-591. [PubMed: 21220299]

8. Williams SS, Retterer S, Lopez R, Ruiz R, Samulski ET, DeSimone JM. Nano Lett. 2010; 10:14211428. [PubMed: 20178369]

9. Knop K, Hoogenboom R, Fischer D, Schubert US. Angew. Chem., Int. Ed. 2010; 49:6288-6308.

10. Gref R, Luck M, Quellec P, Marchand M, Dellacherie E, Harnisch S, Blunk T, Muller RH. Colloids Surf. A. 2000; 18:301-313.

11. Perrault SD, Walkey C, Jennings T, Fischer HC, Chan WCW. Nano Lett. 2009; 9:1909-1915. [PubMed: 19344179]

12. Kamaly N, Xiao ZY, Valencia PM, Radovic-Moreno AF, Farokhzad OC. Chem. Soc. Rev. 2012; 41:2971-3010. [PubMed: 22388185]

13. Hrkach J, Von Hoff D, Ali MM, Andrianova E, Auer J, Campbell T, De Witt D, Figa M, Figueiredo M, Horhota A, Low S, McDonnell K, Peeke E, Retnarajan B, Sabnis A, Schnipper E, Song JJ, Song YH, Summa J, Tompsett D, Troiano G, Hoven TV, Wright J, LoRusso P, Kantoff PW, Bander NH, Sweeney C, Farokhzad OC, Langer R, Zale S. Sci. Transl. Med. 2012; 4:1-11.

14. Chauhan VP, Stylianopoulos T, Boucher Y, Jain RK. Annu. Rev. Chem. Biomol. Eng. 2011; 2:281-298. [PubMed: 22432620]

15. Perry JL, Reuter KG, Kai MP, Herlihy KP, Jones SW, Luft JC, Napier M, Bear JE, DeSimone JM. Nano Lett. 2012; 12:5304-5310. [PubMed: 22920324]

16. Wang J, Tian S, Petros RA, Napier ME, DeSimone JM. J. Am. Chem. Soc. 2010; 132:1130611313. [PubMed: 20698697]

17. Petros RA, Ropp PA, DeSimone JM. J. Am. Chem. Soc. 2008; 130:5008-5009. [PubMed: 18355010]

18. Enlow EM, Luft JC, Napier ME, DeSimone JM. Nano Lett. 2011; 11:808-813. [PubMed: 21265552]

19. Parrott MC, Finniss M, Luft JC, Pandya A, Gullapalli A, Napier ME, DeSimone JM. J. Am. Chem. Soc. 2012; 134:7978-7982. [PubMed: 22545784]

20. Hasan W, Chu K, Gullapalli A, Dunn SS, Enlow EM, Luft JC, Tian S, Napier ME, Pohlhaus PD, Rolland JP, DeSimone JM. Nano Lett. 2011; 12:287-292. [PubMed: 22165988]

21. Dunn SS, Tian S, Blake S, Wang J, Galloway AL, Murphy A, Pohlhaus PD, Rolland JP, Napier ME, DeSimone JM. J. Am. Chem. Soc. 2012; 134:7423-7430. [PubMed: 22475061]

22. Xu J, Wang J, Luft JC, Tian S, Owens G, Pandya AA, Berglund P, Pohlhaus P, Maynor BW, Smith J, Hubby B, Napier ME, DeSimone JM. J. Am. Chem. Soc. 2012; 134:8774-8777. [PubMed: 22568387]

23. Chen K, Merkel TJ, Pandya A, Napier ME, Luft JC, Daniel W, Sheiko S, DeSimone JM. Biomacromolecules. 2012; 13:2748-2759. [PubMed: 22852860] 
A

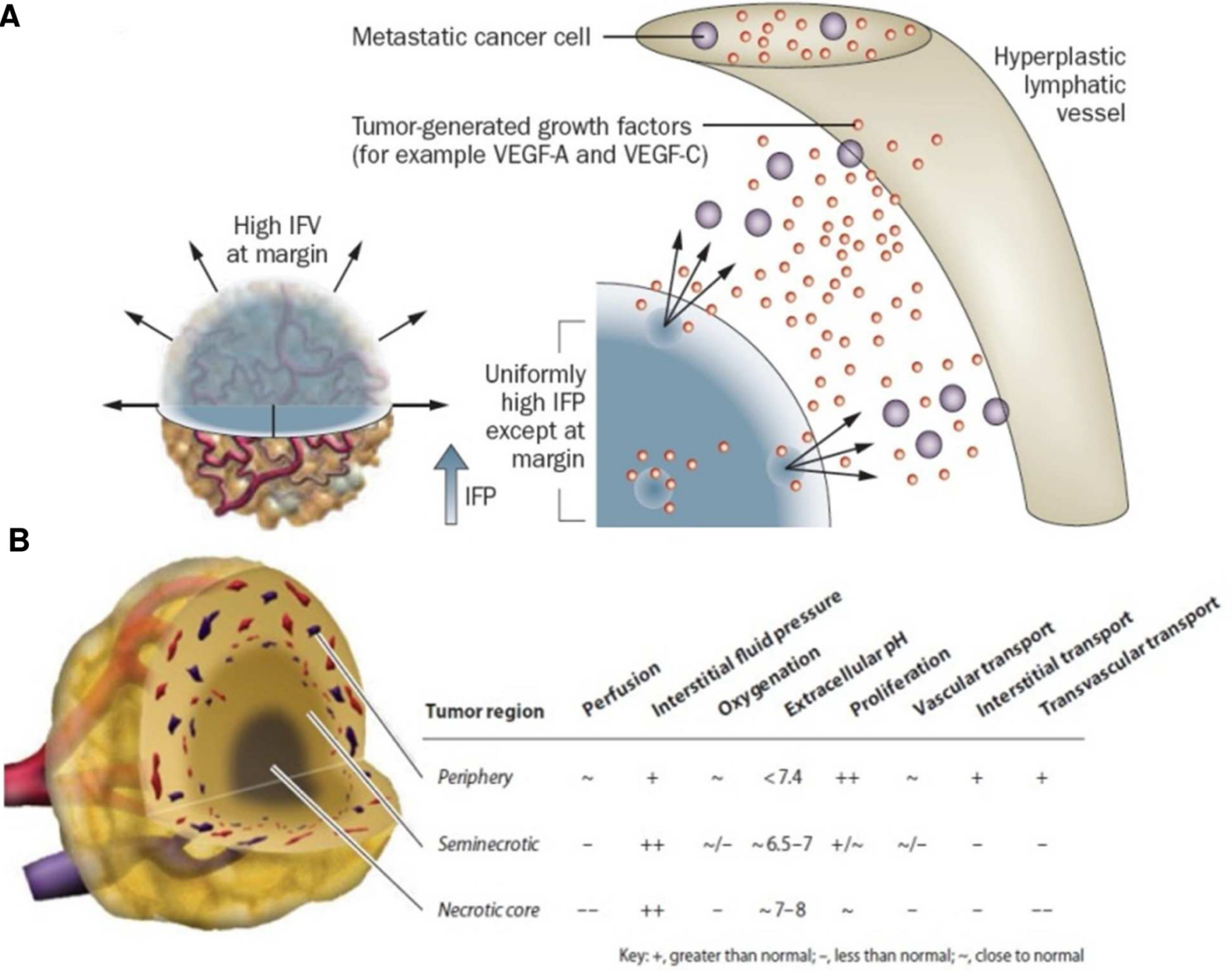

Figure 1.

(A) The IFP is uniformly elevated in tumors except at the margin. The steep drop in IFP at the margin causes fluid, growth factors and cells to leak out of the tumor into the peritumoral tissue, which in turn might facilitate angiogenesis and metastasis, and inhibit drug delivery; ${ }^{1}$ (B) General properties of the tumor microenvironment, including heterogeneous drug delivery. Three regions are present in many tumor types - the periphery, seminecrotic region, and necrotic core. ${ }^{14}$ Adapted with permission from Nature Publishing Group and Annual Reviews. 


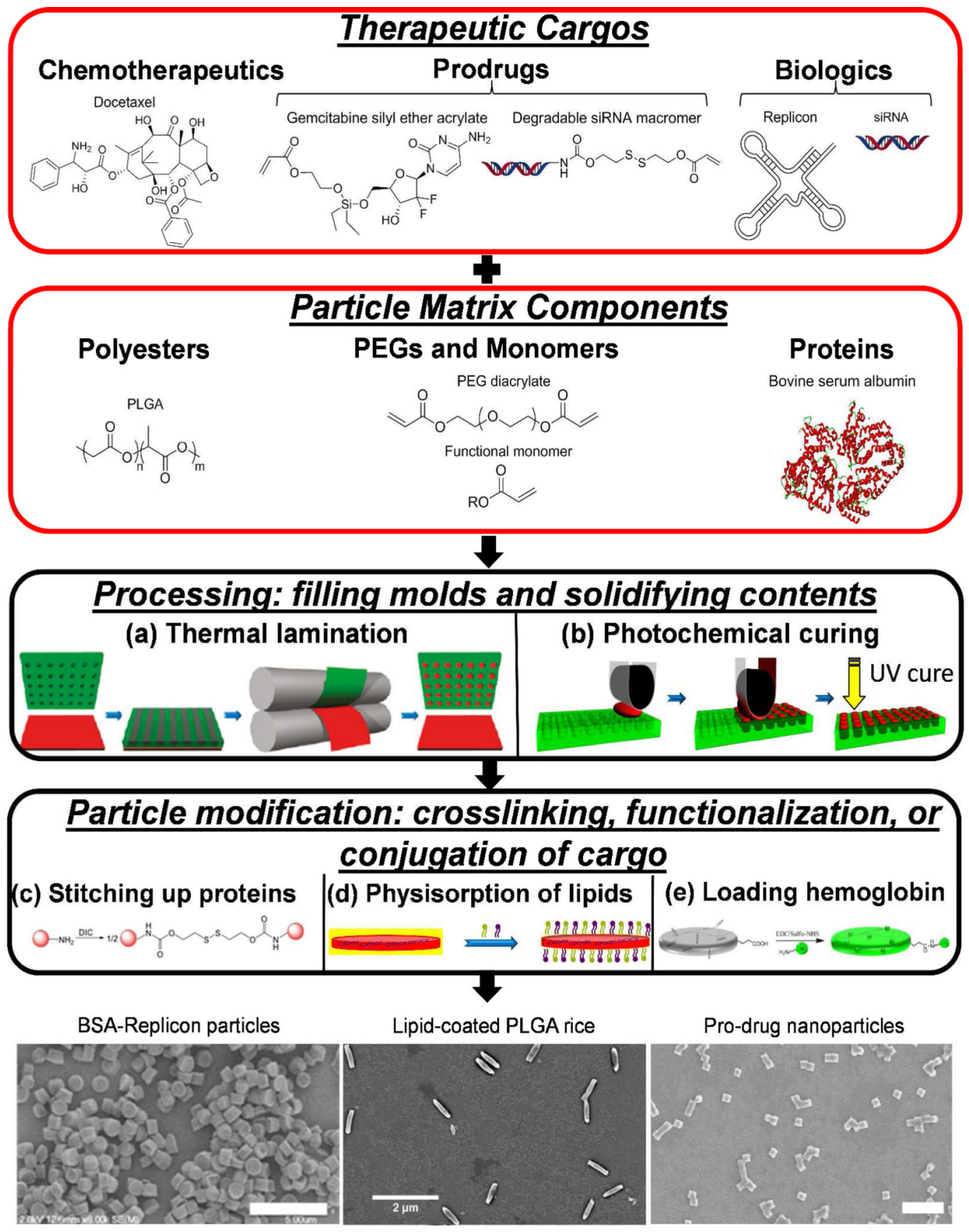

Figure 2.

Examples of therapeutic cargos and particle matrix components that may be mixed and processed into PRINT molds to produce monodisperse, shape-specific particles. (a) A preparticle film (red) is married to PRINT molds (green) at elevated temperature followed by solidification after cooling to room temperature. (b) Pre-particle solution (red) is laminated into PRINT molds (green) followed by exposure to UV light for photochemical curing. (c) Protein (red sphere) contains primary amine residues that are reacted with a disulfidecontaining bis(imidazole carboxylate) (DIC) crosslinker. (d) PLGA-siRNA NPs on harvesting layer of poly(vinyl alcohol) (PVA) (yellow) are collected in solution with cationic and endosomolytic lipids. (e) Lysine residues on hemoglobin are coupled to 
carboxylic acid-containing hydrogel microparticles through amidation. SEM micrographs illustrate the morphology of DIC-crosslinked BSA-Replicon microparticles (scale bar $=5$ $\mu \mathrm{m}$ ), lipid-coated, rod-shaped PLGA particles (scale bar $=2 \mu \mathrm{m}$ ), and gemcitabine prodrug PEG NPs (scale bar $=1 \mu \mathrm{m}$ ). ${ }^{19-20,22}$ Adapted with permission from the American Chemical Society. Copyright 2011 and 2012 American Chemical Society. 